\title{
Le contrôle de la translocation nucléaire des protéines : un niveau supplémentaire de régulation de l'activité des gènes
}

La redistribution intracellulaire de populations entières de protéines lors de chocs thermiques et au cours des stades précoces du développement embryonnaire est une observation assez ancienne qui avait permis d'introduire l'hypothèse selon laquelle le contrôle du transport des protéines vers leur espace fonctionnel pouvait participer à la modulation de l'activité des gènes [1]. Cette notion a récemment été confortée en ce qui concerne le franchissement de l'enveloppe nucléaire par des protéines impliquées dans des fonctions aussi variées que les contrôles de la morphogenèse, de la prolifération cellulaire et de la transduction de signaux hormonaux. Les quelques situations, non exhaustives, décrites ci-dessous convaincront à la fois de l'importance et de la richesse de ce mode de régulation.

Deux premiers exemples spectaculaires concernent l'expression des protéines "Dorsal " et c-Myc dans les phases initiales du développement embryonnaire chez la drosophile et le xénope.

Le produit du gène dorsal, ou $d l$, est un morphogène clé pour la formation de l'asymétrie dorso-ventrale de l'embryon de Drosophila melanogaster. Différents arguments génétiques et biochimiques, ainsi que sa forte homologie avec les proto-oncogènes $c$ rel murin et aviaire, suggèrent qu'il est un facteur transcriptionnel et que, par conséquent, il assure sa fonction dans le noyau. Récemment, trois groupes indépendants ont rapporté que sa redistribution intracellulaire au cours de l'embryogenèse précoce est corrélée à son activation fonctionnelle [2-4]. En résumé, cette protéine est initialement exprimée et répartie de façon homogène dans le cytoplasme de l'œuf non fertilisé. Elle persiste ensuite dans cette localisation diffuse pendant les premières phases de division nucléaire puis se concentre progressivement dans la partie ventrale de l'embryon en établissant donc un gradient vertical. Elle ne pénètre enfin dans les noyaux du blastoderme syncytial qu'à partir de la dixième division nucléaire ; c'està-dire le stade précis où elle endosse son rôle véritable de morphogène et d'effecteur transcriptionnel.

Le produit du proto-oncogène $c-m y c$ est connu pour être essentiellement exprimé dans les cellules somatiques qui prolifèrent. C'est une phosphoprotéine à demi-vie courte qui a toujours été observée dans le noyau cellulaire. Son rôle est encore débattu puisqu'il a été proposé, sans exclusive, qu'elle agisse comme facteur transcriptionnel, comme modulateur post-transcriptionnel ou encore comme régulateur de la réplication. Récemment, Gusse et al., dans le laboratoire de M. Méchali (Institut Jacques-Monod, Paris), [5] ont décrit une série d'observations associant, comme pour $d l$, redistribution subcellulaire et activation fonctionnelle de cette protéine. Le produit c-Myc est exprimé à une concentration extrêmement forte dans le cytoplasme de l'ovocyte quiescent de Xenopus laevis sous forme de protéine d'origine maternelle, particulièrement stable et non phosphorylée. Sitôt la fertilisation effectuée, elle est transportée dans le noyau de l'ovocyte, qui entame une série de divisions extrêmement rapides. Ce transport est associé à la fois à la phosphorylation de la protéine et à l'activation de sa dégradation. Cependant, aucune relation de cause à effet n'a encore pu être démontrée. A la fois du fait des divisions nucléaires, qui entraînent la dilution de la protéine c-Myc maternelle, et du fait de l'activation de la protéolyse, le contenu par noyau de protéine c-Myc décline rapidement pour devenir très faible en fin de phase de segmentation rapide. L'ensemble de ces observations suggère que la vitesse de segmentation de l'œuf de xénope est dépendante de l'abondance de la protéine c-Myc et que le transport de celle-ci dans le noyau à une concentration saturante est un événement clé pour le déclenchement du processus. 
Le cas du bFGF, ou basic fibroblast growth factor, est surprenant à plus d'un égard: si la plupart des cytokines voient leur effet totalement relayé par des médiateurs membranaires et intracellulaires, le bFGF peut, quant à lui, prolonger son action après internalisation dans la cellule. En effet, il est susceptible de se concentrer dans le nucléole, où il stimule la transcription des cistrons ribosomiques. Récemment, Baldin et al. (équipe de F. Amalric, centre de biochimie et de génétique cellulaire, Toulouse) [6] ont montré que la localisation subcellulaire du bFGF dans les cellulaires endothéliales d'aorte de bœuf est dépendante de l'état de prolifération. Si ce facteur de croissance est capable de pénétrer dans le cytoplasme quelle que soit la phase du cycle cellulaire, son entrée dans le noyau est limitée à la phase G1 tardive, permettant ainsi la régulation temporelle fine de son activité stimulatrice de la synthèse des $A R N$ ribosomiques.

Certaines protéines pourraient avoir un rôle de messager intracellulaire permettant d'ajuster le métabolisme nucléaire au métabolisme cytoplasmique et vice versa. C'est ce que tendent à montrer les travaux récents de Borer et al. [7]. La nucléoline et la protéine $\mathrm{B} 23 / \mathrm{n}^{\circ} 38$ sont des protéines nucléolaires majeures considérées, il y a peu de temps encore, comme exclusivement nucléaires et dont les fonctions seraient de contrôler les premières étapes de la synthèse des ARN ribosomiques et l'assemblage des pré-ribosomes. En utilisant d'élégantes techniques de micro-injection et de fusion cellulaire, ces auteurs ont montré qu'elles effectuent, en réalité, une navette permanente entre noyau et cytoplasme et pourraient, de ce fait, être impliquées dans le transport des constituants ribosomiques. En outre, le jeu de phosphorylations complexes auxquelles elles sont assujetties soulève l'hypothèse d'un lien régulationnel entre la demande cytoplasmique et la production nucléaire des ribosomes par le biais des modifications post-traductionnelles intervenant dans le cytoplasme.

La notion selon laquelle certains facteurs transcriptionnels sont stockés sous forme latente dans le cyto-

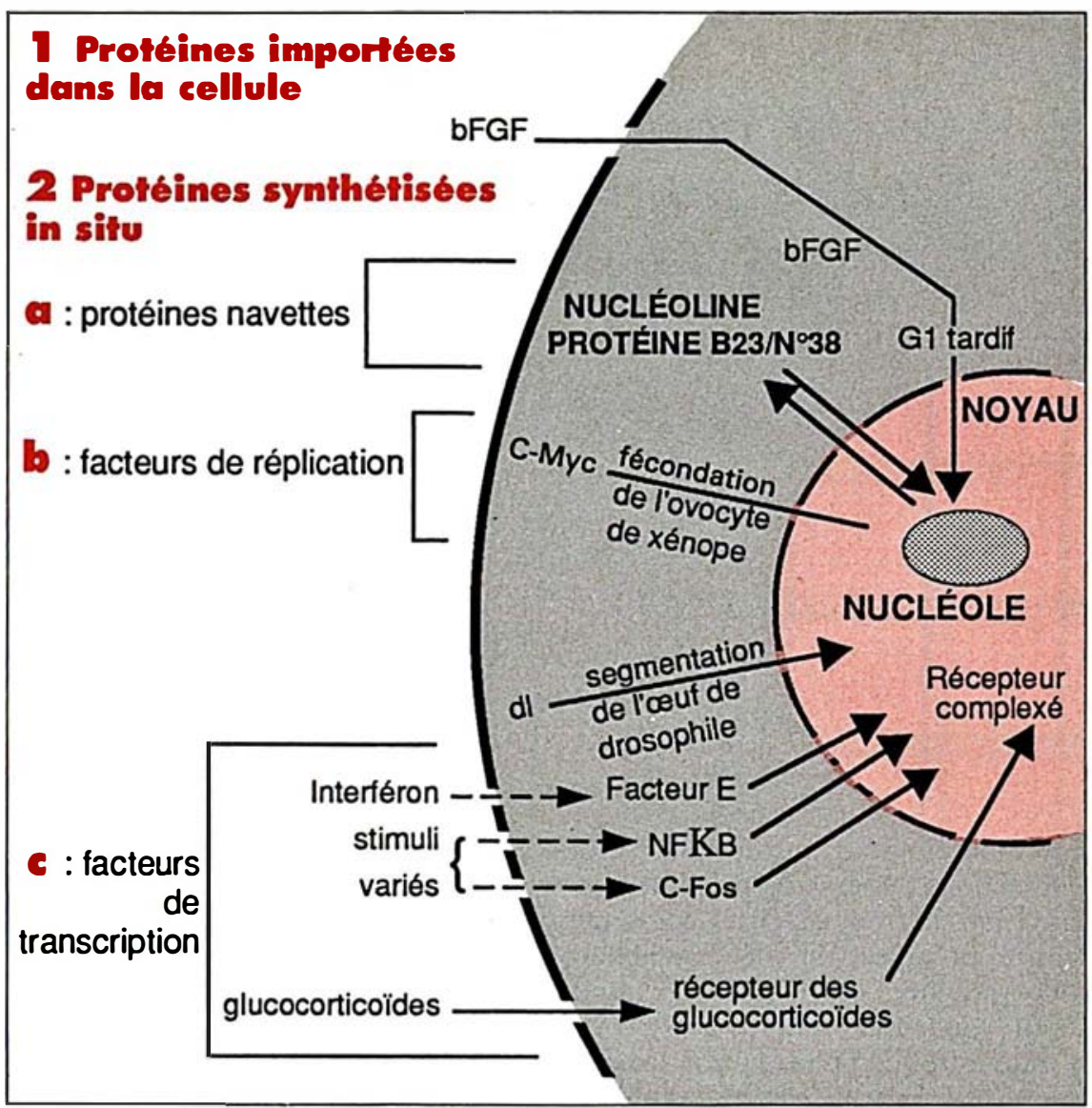

Figure 1. Deux grandes classes de protéines dont le transport dans le noyau est contrôlé peuvent être distinguées : (1) Les protéines importées dans la cellule à partir du milieu extra-cellulaire. Le bFGF en est un exemple puisqu'il ne pénètre dans le noyau qu'en phase G1 tardive. (2) Les protéines synthétisées dans la cellule même. Trois sous-groupes peuvent à nouveau être distingués. II s'agit : (a) des protéines navettes qui ajustent les métabolismes cytoplasmique et nucléaire. Elles sont représentées par la nucléoline et la protéine $B 23 / N^{\circ} 38$, (b) certains facteurs de réplication comme la protéine $c-M y c$ de Xénope dont le transport du cytoplasme dans le noyau de l'ovocyte est activé par la fécondation, (c) les facteurs transcriptionnels latents. Leur translocation cyto-nucléaire peut dépendre de la fixation d'un ligand à la membrane cellulaire (NF $\kappa B$, facteur $E, c$-Fos) ou de la pénétration d'un "ligand activateur " spécifique (récepteur aux glucocorticoïdes). Dans chaque situation, ni les cascades métaboliques impliquées, ni l'intervention du cytosquelette, ni le mécanisme de franchissement de l'enveloppe nucléaire ne sont connus.

plasme, puis activés par translocation dans le noyau à la suite de stimuli appropriés, se renforce régulièrement. Les cas de $\mathrm{NF}_{\kappa} \mathrm{B}$ et du récepteur aux glucocorticoïdes sont certainement les mieux documentés. Bien qu'ayant été initialement caractérisé comme stimulateur transcriptionnel des gènes d'immunoglobulines $\kappa, \mathrm{NF} \kappa \mathrm{B}$ est maintenant connu pour être un facteur de transcription ubiquitaire responsable de l'activation d'une grande variété de gènes incluant ceux codant pour différentes cytokines et leurs récepteurs, les antigènes d'histocompatibilité et leurs protéines associées ainsi que certains virus. Dans les lymphocytes $B$ et $T$, de même que 
dans les monocytes différenciés, son activité de liaison à l'ADN est constitutive. En revanche, dans de nombreux autres types cellulaires, elle n'est pas détectable mais est inductible, souvent de façon transitoire, par des agents très divers comme le TPA (un ester de phorbol), le LPS (lipopolysaccharide d'origine bactérienne), des lectines, l'ARN double brin, l'interleukine 1, le $\mathrm{TNF} \alpha$ et l'AMPc (pour revue : [8]). La sousunité de $\mathrm{NF}_{\kappa} \mathrm{B}$ interagissant directement avec l'ADN est un polypeptide de $50 \mathrm{kDa}$ formant des homodimères stables. Une protéine de $65 \mathrm{kDa}$, n'ayant pas d'affinité pour l'ADN, est systématiquement associée à la p50 activée. Les magnifiques expériences de Bauerle et al. (pour revue : [8]), ont montré que $\mathrm{NF} \kappa \mathrm{B}$ inactif est localisé dans le cytoplasme où il est complexé à un inhibiteur protéique (deux formes ont déjà été caractérisées : I $\kappa \mathrm{B} \alpha$ et $\mathrm{I} \kappa \mathrm{B} \beta$ ) dont la fonction est double, puisqu'il empêche à la fois le transport du facteur dans le noyau in vivo et la liaison aux séquences nucléiques cibles in vitro. En réponse à l'agent inducteur, le complexe $\mathrm{NF}_{\kappa} \mathrm{B}-\mathrm{I}_{\kappa} \mathrm{B}$ se dissocie pour permettre à $\mathrm{NF}_{\kappa} \mathrm{B}$ de pénétrer dans le noyau et d'y assumer son rôle. L'activation fonctionnelle s'opère en absence de synthèse protéique de novo suggérant que des modifications posttraductionnelles de $\mathrm{NF}_{\kappa} \mathrm{B}$ et/ou de I $\mathrm{B}$ pourraient être impliquées. Récemment, il a été montré que $\mathrm{I} \kappa \mathrm{B}$, en sus de sa capacité à inhiber leur formation, est aussi capable de dissocier in vitro les complexes $\mathrm{NF}_{\kappa} \mathrm{B}$ $\mathrm{ADN}$. Cette observation amène à penser qu'in vivo $\mathrm{I} \kappa \mathrm{B}$ pourrait intervenir non seulement comme inhibiteur du transport nucléaire, mais aussi pour inactiver $\mathrm{NF}_{\kappa} \mathrm{B}$ dans le noyau et le ramener dans le cytoplasme. Cette hypothèse permet, en particulier, de rendre compte de l'activation transitoire de $\mathrm{NF} \kappa \mathrm{B}$ observée dans différentes situations : $\mathrm{NF} \kappa \mathrm{B}$ étant une protéine relativement stable, la disparition de son activité de liaison à l'ADN dans le noyau ne peut pas être expliquée par une dégradation protéolytique, mais seulement par le retour du facteur $\mathrm{m} / \mathrm{s} n^{\circ} 8$, vol. 6 , octobre 90 transcriptionnel dans le cytoplasme. Le récepteur des glucocorticoïdes est une protéine intracellulaire. Il ne transduit le signal hormonal qu'après liaison physique de son ligand en s'associant à des séquences d'ADN particulières, appelées GRE (glucocorticoid response elements), pour stimuler la transcription de gènes spécifiques. Picard et Yamamoto [9] ont montré que le récepteur sans son ligand s'accumule dans le cytoplasme, où il fait partie intégrante d'un complexe multiprotéique qui pourrait avoir une fonction d'ancrage et dont l'un des constituants serait la protéine de choc thermique hsp 90. Suite à la liaison de l'hormone, le complexe récepteurligand est rapidement libéré sous forme de monomère capable de pénétrer dans le noyau. Le contrôle du transport est assuré par au moins deux motifs internes au récepteur dont l'activité caryophile est strictement hormono-dépendante. Il est cependant intéressant de remarquer que la pénétration dans le noyau et la fixation à l'ADN peuvent être découplées, suggérant deux étapes successives dans la voie de transduction du signal hormonal.

Sans avoir été analysés au niveau moléculaire avec autant de détails, les médiateurs de la réponse aux interférons et le proto-oncogène $c$-fos sont aussi informatifs. Les interférons, en se liant à leurs récepteurs membranaires sur les cellules cibles, induisent l'expression d'au moins une centaine de gènes. Les régions promotrices de plusieurs d'entre eux contiennent des séquences caractéristiques (ISRE ou interferon stimulated regulatory elements) reconnues par des protéines spécifiques (facteurs E) lorsque les cellules sont stimulées par un interféron. Dale $e t$ al. [10] ont récemment montré, à l'image de $\mathrm{NF}_{\kappa} \mathrm{B}$, que le facteur $\mathrm{E}$ est présent dans le cytoplasme de cellules incubées en absence d'interféron. En revanche, il est transporté dans le noyau, en moins d'une minute et sans synthèse protéique de novo, en réponse à l'addition d'interféron $\alpha$ et devient ainsi capable de se lier aux motifs ISRE. Le proto-oncogène $c$-fos code pour un facteur transcriptionnel. Il est exprimé à bas niveau dans la plupart des cellules et est assujetti à une régulation transcriptionnelle et posttranscriptionnelle sévère et extrêmement complexe. En effet, son expression constitutive, même à bas niveau, est suffisante pour conférer aux cellules des propriétés transformantes. Il est en général induit rapidement et transitoirement par des stimuli variés dans des contextes cellulaires extrêmement divers. Sa fonction biologique est encore inconnue, mais il est admis qu'il agirait comme un " commutateur général " ubiquitaire, nécessaire aux cellules pour transformer des signaux à court terme en réponses à long terme comme la division cellulaire, la différenciation et même la formation de la mémoire. Bien que la protéine c-Fos ait essentiellement été rapportée comme étant nucléaire, nous venons de montrer qu'il est possible de dissocier sa synthèse de son transport dans le noyau [11]. Couplées aux observations récentes montrant qu'elle pouvait être retenue dans le cytoplasme de kératinocytes ainsi que dans celui de cellules de la surrénale, nos expériences suggèrent, comme pour $\mathrm{NF}_{\kappa} \mathrm{B}$ et le facteur $\mathrm{E}$, que le transport de la protéine c-Fos est sous la dépendance d'une stimulation extracellulaire permanente, probablement différente suivant le type cellulaire concerné. L'effet de cette stimulation peut être relayé au niveau intracellulaire par la protéine kinase dépendante de l'AMP cyclique et par un inhibiteur protéique à durée de vie courte, rappelant ainsi le cas de $\mathrm{NF}_{\kappa} \mathrm{B}$. De façon intéressante, le proto-oncogène c-fos a été transduit sous forme mutée par deux rétrovirus oncogènes murins. Nous avons montré que les protéines virales Fos migrent constitutivement dans le noyau dans des conditions où leur homologue cellulaire est retenu dans le cytoplasme, mettant ainsi en évidence la possibilité d'une contribution nouvelle au pouvoir tumorigène d'un oncogène. Bien qu'en étant l'image miroir, cette situation est à rapprocher de celle de l'oncogène $v$ $a b l$, dont l'effet transformant est dépendant de la localisation anormale 
de son produit protéique : en raison des altérations structurales engendrées lors de la transduction rétrovirale, la protéine de fusion Gag-Abl du virus de la leucémie murine d'Abelson manifeste constitutivement une activité tyrosine kinase et, contrairement à son homologue cellulaire, qui est essentiellement nucléaire, s'accumule dans le cytoplasme et s'associe à la membrane cellulaire, changeant probablement ainsi la nature de ses substrats [12].

En conclusion, le transport des protéines au travers de l'enveloppe nucléaire, dans un sens et dans l'autre, est une voie importante de transduction de signaux utilisée dans des situations physiologiques très diverses. Les exemples sélectionnés ci-dessus montrent déjà que les mécanismes mis en jeu pour contrôler ces flux migratoires sont à la fois variés et complexes. Leur étude constitue l'un des champs exploratoires les plus récents de la biologie cellulaire.
Marc Piechaczyk, Pierre Roux

Laboratoire de biologie moléculaire, URA Cnrs 1191 "Génétique moléculaire ", USTL, place E.-Bataillon, 34095 Montpellier Cedex 5, France.

\section{RÉFÉRENCES}

1. Dingwall C, Laskey RA. Protein import into the cell nucleus. Ann Rev Cell Biol 1986 ; 2 : 367-90.

2. Roth S, Stein D, Nusslein-Volhard C A gradient of nuclear localization of the dorsal protein determines dorso-ventral pattern in the drosophila embryo. Cell 1989 ; 59 : 1189-202.

3. Rushlow GA, Han K, Manley JL, Levine $\mathrm{A}$. The graded distribution of the dorsal morphogen is initiated by selective nuclear transport in drosophila. Cell 1989 ; 59: 1165-75.

4. Steward R. Relocalization of the dorsal protein from the cytoplasm to the nucleus correlates with its function. Cell $1989 ; 59$. 1179-88.

5. Gusse M, Ghysdael J, Evan G, Soussi T, Mechali M. Translocation of a store of maternal cytoplasmic c-Myc protein into nuclei during Xenopus early development. Mol Cell Biol 1989 ; 9 : 5395-403.
6. Baldin V, Roman AM, Bosc-Bierne I, Amalric F. Bouche G. Translocation of bFGF to the nucleus in G1 phase cell cycle specific in bovine aortic endothelial cells. EMBO J 1990 ; 9 : 1511-8.

7. Borer RA, Lehner CF, Eppenberger HM, Nigg EA. Major nucleolar proteins shuttle between nucleus and cytoplasm. Cell $1989 ; 56: 379-90$

8. Lenardo $\mathrm{ML}$, Baltimore D. $\mathrm{NF} \kappa \mathrm{B}$ : a pleiotropic mediator of inducible and tissuespecific gene control. Cell $1989 ; 58$ : 227-9. 9. Picard D, Yamamoto KY. Two signals mediate hormone-dependent nuclear localization of the glucocorticoid receptor. $E M B O$ J 1987 ; 6 : 3333-40.

10. Dale TC, Ali-Imam AM, Kerr IM, Stark GP. Rapid activation by interferon $\alpha$ of a latent DNA-binding protein present in the cytoplasm of untreated cells. Proc Natl Acad Sci USA 1989 ; 86 : 1203-7.

11. Roux P, Blanchard J-M, Fernandez A, Lamb N, Jeanteur P, Piechaczyk $M$. Nuclear localization of c-Fos, but not v-Fos proteins, is dependent upon extra-cellular stimulation. Cell (sous presse).

12. Van Etten RA, Jackson P, Baltimore $\mathrm{D}$. The mouse type IV $c$-abl gene product is a nuclear protein and activation of its transforming activity is associated with cytoplasmic localization. Cell 1989 ; 58 : 669-78. 